The Astrophysical Journal, 490:368-376, 1997 November 20

(C) 1997. The American Astronomical Society. All rights reserved. Printed in U.S.A.

\title{
SPECTRAL ENERGY DISTRIBUTIONS OF T TAURI STARS WITH PASSIVE CIRCUMSTELLAR DISKS
}

\author{
E. I. Chitang AND P. GoldReich \\ California Institute of Technology, Pasadena, CA 91125; echiang@tapir.caltech.edu,pmg@nicholas.caltech.edu \\ Received 1997 March 18; accepted 1997 July 3
}

\begin{abstract}
We derive hydrostatic, radiative equilibrium models for passive disks surrounding $T$ Tauri stars. Each disk is encased by an optically thin layer of superheated dust grains. This layer reemits directly to space about half the stellar energy it absorbs. The other half is emitted inward and regulates the interior temperature of the disk. The heated disk flares. As a consequence, it absorbs more stellar radiation, especially at large radii, than a flat disk would. The portion of the spectral energy distribution contributed by the disk is fairly flat throughout the thermal infrared. At fixed frequency, the contribution from the surface layer exceeds that from the interior by about a factor 3 and is emitted at more than an order of magnitude greater radius. Spectral features from dust grains in the superheated layer appear in emission if the disk is viewed nearly face-on.
\end{abstract}

Subject headings: accretion, accretion disks - circumstellar matter - infrared: stars radiative transfer - stars: pre-main-sequence

\section{INTRODUCTION}

Excess infrared (IR) emission from $T$ Tauri stars is thought to arise from circumstellar disks (Mendoza 1968; Shu, Adams, \& Lizano 1987; Adams, Lada, \& Shu 1987; and references therein). The standard disk is modeled as a flat blackbody. In the simplest scenario, it lacks intrinsic luminosity and passively reradiates the energy it absorbs from the central star. This blackbody model yields an infrared spectral energy distribution (SED) of the form $v F_{v} \propto v^{n}$, with spectral index $n=4 / 3$ (Adams et al. 1987 and references therein). By coincidence, the steady state accretional luminosity from an opaque, thin disk yields an SED with identical spectral index (Lynden-Bell \& Pringle 1974). SEDs measured between 3 and $100 \mu \mathrm{m}$ are usually well fitted by power laws with $0 \leq n \leq 4 / 3$. However, in contrast to the prediction of the standard model, most sources exhibit flattish spectra having $n \leq 3 / 4$ (see, e.g., Rydgren et al. 1984; Rucinski 1985; Rydgren \& Zak 1987; Strom et al. 1989; Beckwith et al. 1990).

The failure of the standard disk model spawned a number of alternative proposals. Of greatest relevance here is the investigation by Kenyon \& Hartmann (1987) of a blackbody disk whose surface flares outward with increasing radius as a consequence of vertical hydrostatic equilibrium. Flared disks intercept more stellar radiation than flat ones, especially at large distances from the star. Other models invoke either an "active" disk having a high intrinsic luminosity or a dusty component in addition to the disk. The dissipation of a one-armed spiral density wave as an intrinsic disk heating mechanism falls in the first category (Adams, Ruden, \& Shu 1989; Shu et al. 1990; Ostriker, Shu, \& Adams 1992). The second category includes the proposal that a tenuous dusty envelope surrounds the star/disk system and either scatters stellar radiation back onto the disk (Natta 1993) or absorbs and reemits it in the infrared (Calvet et al. 1994).

We calculate the SED for the passive, reprocessing disk in a self-consistent fashion. The basic features and results of our investigation are set forth in $\S 2$. Extensions and refinements are considered in $\S 3$. Lastly, in $\S 4$, we discuss our results in the context of observations and summarize unresolved issues.

\section{THEORY}

\subsection{Model Assumptions}

We consider a $\mathrm{T}$ Tauri star surrounded by a passive disk. The star is modeled as a spherical blackbody of temperature $T_{*}=4000 \mathrm{~K}$, mass $M_{*}=0.5 M_{\odot}$, and radius $R_{*}=2.5 R_{\odot}$ (see, e.g., Table II in Beckwith et al. 1990). Our fiducial disk has a surface mass density similar to that of the minimummass solar nebula, $\Sigma=a_{\mathrm{AU}}^{-3 / 2} \Sigma_{0}$, where $a_{\mathrm{AU}}$ is the disk radius measured in astronomical units and $\Sigma_{0}=10^{3} \mathrm{~g}$ $\mathrm{cm}^{-2}$ (Weidenschilling 1977). Dust, which is uniformly mixed with the gas, comprises about $1 \%$ of the total mass. Dust grains dominate the continuum opacity from visible through millimeter wavelengths. We take the grains to be spheres with radius $r=0.1 \mu \mathrm{m}$, mass density $\rho_{d}=2 \mathrm{~g} \mathrm{~cm}^{-3}$, and negligible albedo. Their emissivity, which is unity for $\lambda \leq 2 \pi r$, decreases as $\epsilon_{\lambda} \approx(2 \pi r / \lambda)^{\beta}$ for $\lambda \geq 2 \pi r$. We denote by $\epsilon \approx(8 \pi r k T / h c)^{\beta} \approx\left(T / T_{*}\right)^{\beta}$ the average dust emissivity at temperature $T$. For our fiducial disk, $\beta=1$. The dust opacity at visual wavelengths is $\kappa_{V} \approx 400 \mathrm{~cm}^{2} \mathrm{~g}^{-1}$, which implies an optical depth $\tau_{V} \approx 4 \times 10^{5} a_{\mathrm{AU}}^{-3 / 2}$ for our fiducial disk.

SEDs are computed for disks viewed pole-on. We choose an inner cutoff radius, $a_{i} \approx 6 R_{*} \approx 0.07 \mathrm{AU}$, to mark the condensation boundary of common silicates. The outer cutoff radius for flat disks, $a_{o} \approx 2.3 \times 10^{4} R_{*} \approx 2.7$ $\times 10^{2} \mathrm{AU}$, is fixed to facilitate comparisons among different disk models; $a_{o}$ is comparable to the size of the largest disks seen in silhouette against the Orion Nebula (McCaughrean \& O’Dell 1996).

Our model assumptions are chosen to make the telling of our story as simple and direct as possible. Issues regarding these choices and possible alternatives are dealt with in $\S 3$.

\subsection{Blackbody Disk}

To begin, we review standard relations for the temperature and SED of a blackbody disk. The flux of stellar 


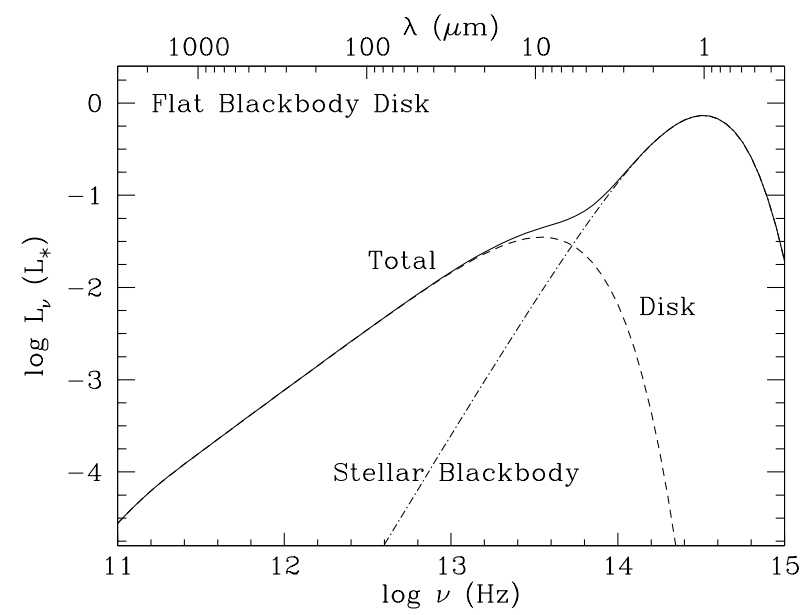

FIG. 1.-SED for the flat blackbody disk, with contributions from star and disk identified. The $n=4 / 3$ law is evident between $30 \mu \mathrm{m}$ and $1 \mathrm{~mm}$. The turnover near $1 \mathrm{~mm}$ is due to our truncation of the disk at $a_{o} \approx 270$ AU.

radiation incident upon the disk is $(\alpha / 2)\left(R_{*} / a\right)^{2} \sigma T_{*}^{4}$ for $a \gg$ $R_{*}$, where $\alpha$ is the grazing angle at which the starlight strikes the disk. Equating the emitted and absorbed fluxes yields the disk temperature ${ }^{1}$

$$
T_{e} \approx\left(\frac{\alpha}{2}\right)^{1 / 4}\left(\frac{R_{*}}{a}\right)^{1 / 2} T_{*}
$$

The SED is computed as

$$
L_{v} \equiv 4 \pi d^{2} v F_{v}=8 \pi^{2} v \int_{a_{i}}^{a_{o}} d a a B_{v}\left(T_{e}\right),
$$

where $B_{v}(T)$ is the Planck function, and $d$ is the distance to the source. A scaling relation for $L_{v}$ at wavelengths between those that characterize the disk at $a_{\mathrm{i}}$ and those that characterize the disk at $a_{o}$ is derived as follows. Equation (2) is approximated as $8 \pi^{2} a^{2} v B_{v}\left(T_{e}\right), v B_{v}\left(T_{e}\right)$ is replaced by $\sigma T_{e}^{4} / \pi$, $a$ is related to $T_{e}$ by equation (1), and $T_{e}$ is expressed in terms of $v$ by $3 k T_{e} \sim h v$. These steps yield

$$
L_{v} \sim 8 \pi a^{2} \sigma T_{e}^{4} \sim \alpha L_{*} .
$$

In other words, the fraction of the stellar luminosity $L_{*}$ that is reprocessed to frequencies in an octave centered on $v$ is approximately equal to the grazing angle $\alpha$ at the location where $3 k T_{e}=h v$.

\subsubsection{Flat Geometry}

A flat disk is one whose aspect ratio (opening angle) is independent of $a$; we assume it to be much less than unity. The grazing angle appropriate to this geometry is $\alpha \approx$ $0.4 R_{*} / a \ll 1$ for $a / R_{*} \gg 1$ (Kusaka, Nakano, \& Hayashi 1970; Ruden \& Pollack 1991). Thus, $T_{e}$ from equation (1) takes the form

$$
T_{e} \approx\left(\frac{2}{3 \pi}\right)^{1 / 4}\left(\frac{R_{*}}{a}\right)^{3 / 4} T_{*} .
$$

Application of the scaling relation given by equation (3) to the flat disk gives $L_{v} \sim 0.01\left(v / 10^{13} \mathrm{~Hz}\right)^{4 / 3} L_{*}$. Figure 1 confirms that $L_{v}$ obeys this relation for $30 \lesssim \lambda \lesssim 1000 \mu \mathrm{m}$.

\subsubsection{Hydrostatic Equilibrium}

We retain the assumption that the disk radiates as a blackbody and consider the consequences of vertical hydro-

\footnotetext{
${ }^{1}$ The subscript $e$ is used to denote effective temperature.
}

static equilibrium in a gravitational field $g=\Omega^{2} z$. This is the case investigated by Kenyon \& Hartmann (1987).

The disk temperature $T_{e}$ is still given by equation (1), but now the grazing angle $\alpha$ takes the more general form

$$
\alpha \approx \frac{0.4 R_{*}}{a}+a \frac{d}{d a}\left(\frac{H}{a}\right),
$$

where $H$ is the height of the visible photosphere above the disk midplane.

Taking the gas to be isothermal at temperature $T_{e}$ yields a Gaussian vertical density profile

$$
\frac{n}{n_{0}}=\exp \left(-\frac{z^{2}}{2 h^{2}}\right)
$$

where

$$
\frac{h}{a}=\left(\frac{T_{e}}{T_{c}}\right)^{1 / 2}\left(\frac{a}{R_{*}}\right)^{1 / 2} .
$$

The temperature $T_{c}$ is a measure of the gravitational potential at the surface of the central star;

$$
T_{c} \equiv \frac{G M_{*} \mu_{g}}{k R_{*}} \approx 8 \times 10^{6} \mathrm{~K},
$$

with $\mu_{g}$ the mean molecular weight of the gas. Under the assumption that the dust-to-gas ratio is uniform throughout the disk, we find

$$
\frac{H}{h}=\left[2 \ln \left(\frac{n_{0}}{n_{\mathrm{ph}}}\right)\right]^{1 / 2},
$$

where $n_{\mathrm{ph}}$ is the number density in the photosphere.

Armed with equations (5)-(9), we are ready to evaluate $H / a$. In the limit of large radius where $\alpha$ is dominated by the flaring term,

$$
\frac{H}{a} \approx 4\left(\frac{T_{*}}{T_{c}}\right)^{4 / 7}\left(\frac{a}{R_{*}}\right)^{2 / 7} \approx 0.18 a_{\mathrm{AU}}^{2 / 7} .
$$

In writing equation (10), we have set $H / h=4$; in reality, this factor declines from about 5 at $a_{\mathrm{AU}}=3$ to 4 at $a_{\mathrm{AU}}=10^{2}$.

It follows from equations (5) and (10) that $\alpha \approx 0.005 a_{\mathrm{AU}}^{-1}$ $+0.05 a_{\mathrm{AU}}^{2 / 7}$. Thus, $\alpha$ is minimal at the transition radius $a_{\mathrm{tr}} \approx$ $0.4 \mathrm{AU}$ with $\alpha_{\min } \approx 0.05$. Beyond the transition radius, the disk flares until $H \approx a$ at $a_{\mathrm{o}} \approx 270 \mathrm{AU}$.

The SED for the flared blackbody disk truncated at $a_{o}$, as computed from equation (2), is shown in Figure 2. At wavelengths between 10 and $100 \mu \mathrm{m}$, the disk emission follows the scaling relation (cf. eq. [3]) $L_{v} \sim \alpha L_{*} \sim$ $0.1\left(v / 10^{13} \mathrm{~Hz}\right)^{-2 / 3} L_{*}$. Longward of $300 \mu \mathrm{m}$ in the RayleighJeans regime, the SED varies as $v^{3}$.

\subsection{Radiative Equilibrium Disk}

Here we drop the blackbody assumption and determine the SED by applying the techniques of radiative transfer. The definition of the effective temperature, $T_{e}$, remains as given by equation (1). A simplified description of the disk distinguishes two regions. The surface layer contains grains that are directly exposed to light from the central star. Variables evaluated there are denoted by a subscript $s$. We allow for the possibility that the gas temperature in this layer, $T_{g s}$, may be smaller than the dust temperature, $T_{d s}$. The rest of the disk is lumped together as the interior. Variables evaluated there carry a subscript $i$. We assume that in steady state, the interior gas and dust temperatures are equal; $T_{g i}=T_{d i}=T_{i}$. 


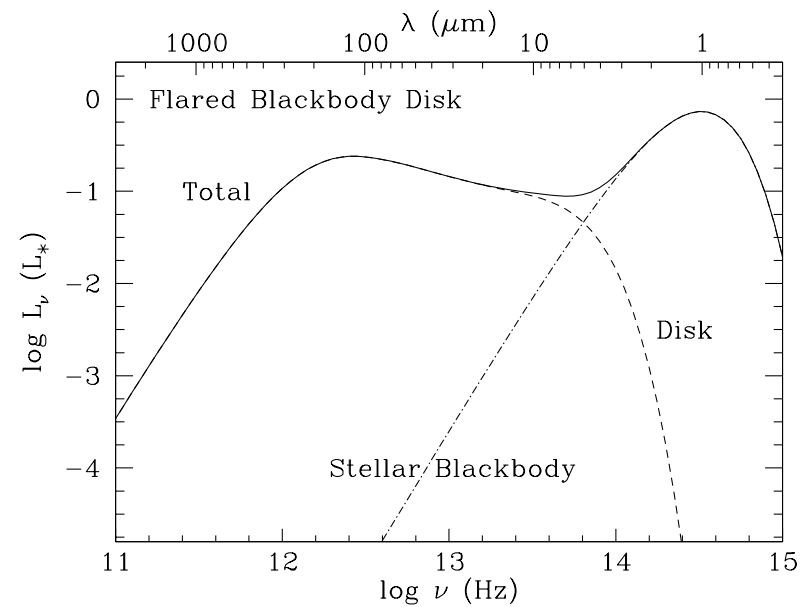

FIG. 2.-SED for the flared blackbody disk. At mid-IR wavelengths, $L_{v} \propto v^{-2 / 3}$. At longer wavelengths, $L_{v} \propto v^{3}$.

A schematic of how the stellar radiation is reprocessed is illustrated in Figure 3. The radiation penetrates the disk to an optical depth of order unity along a slant path inclined by an angle $\alpha \approx 0.4 R_{*} / a$ to the surface. Dust particles along the slant path are "superheated" to a temperature

$$
T_{d s} \approx \frac{1}{\epsilon_{s}^{1 / 4}}\left(\frac{R_{*}}{2 a}\right)^{1 / 2} T_{*} \approx \frac{550}{a_{\mathrm{AU}}^{2 / 5}} \mathrm{~K},
$$

which is greater than the blackbody temperature $T_{\mathrm{BB}} \approx$ $\left(R_{*} / a\right)^{1 / 2} T_{*}$ because their absorptivity in the visible exceeds their emissivity in the infrared. The normal optical depth of the superheated dust layer at visual wavelengths is $\tau_{V} \approx$ $\alpha \approx 0.4 R_{*} / a$. The infrared optical depth is smaller still; $\tau_{\mathrm{IR}} \approx \alpha \epsilon_{s}$.

The superheated dust radiates equal amounts of IR radiation into the inward and outward hemispheres. The outward-directed radiation is similar to that of a dilute blackbody. Where the disk is opaque to blackbody radiation at

$$
T_{i} \approx \frac{T_{e}}{2^{1 / 4}} \approx\left(\frac{\alpha}{4}\right)^{1 / 4}\left(\frac{R_{*}}{a}\right)^{1 / 2} T_{*},
$$

the inward-directed radiation is thermalized at that temperature. The outer boundary of this region is denoted by $a_{\mathrm{th}}$. Just outside $a_{\mathrm{th}}$, the interior is optically thin to its own radiation but still opaque to radiation from the superheated

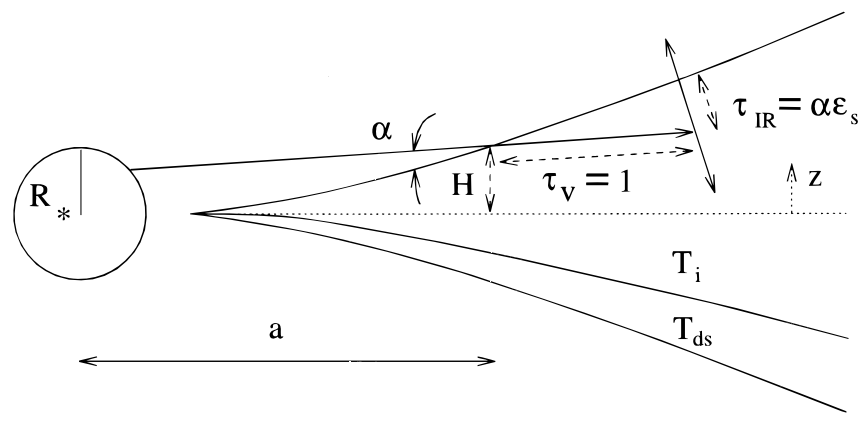

FIG. 3.-Radiative transfer in the passive disk. Stellar radiation strikes the surface at an angle $\alpha$ and is absorbed within visible optical depth unity. Dust particles in this first absorption layer are superheated to a temperature $T_{d s}$. About half of the emission from the superheated layer emerges as dilute blackbody radiation. The remaining half heats the interior to a temperature $T_{i}$. surface; in these regions, the interior temperature is determined by thermal balance to be

$$
T_{i} \approx\left(\frac{\alpha}{4 \epsilon_{i} \kappa_{V} \Sigma}\right)^{1 / 4}\left(\frac{R_{*}}{a}\right)^{1 / 2} T_{*} .
$$

At still greater radii, the encased material is transparent both to its own radiation and to radiation from the surface; the internal temperature here is given by

$$
T_{i} \approx\left(\frac{\alpha \epsilon_{s}^{2}}{\epsilon_{i}}\right)^{1 / 4} T_{d s} \approx\left(\frac{\alpha \epsilon_{s}}{4 \epsilon_{i}}\right)^{1 / 4}\left(\frac{R_{*}}{a}\right)^{1 / 2} T_{*} .
$$
from

The SED for the radiative equilibrium disk is computed

$$
L_{v}=8 \pi^{2} v \int_{a_{i}}^{a_{o}} d a a \int_{-\infty}^{\infty} d z \frac{d \tau_{v}}{d z} e^{-\tau_{v}} B_{v}(T),
$$

where $\tau_{v}$ measures optical depth from $z$ to $\infty$ along the axis perpendicular to the disk midplane.

\subsubsection{Flat Geometry}

Once again, we consider the flat disk, but now under conditions of radiative equilibrium. The appropriate expression for the effective temperature is given by equation (4). For our fiducial flat disk, $a_{\mathrm{th}} \approx 50 \mathrm{AU}$. Runs of $T_{d s}$ and $T_{i}$ as functions of $a$ are displayed in Figure 4 .

The SED for the flat, radiative equilibrium disk as calculated from equation (13) is displayed in Figure 5. Its appearance is similar to that of the SED for the flat blackbody disk. Over most of the IR, it is dominated by radiation from the optically thick interior. The surface layer radiates more than the interior shortward of $6 \mu \mathrm{m}$; however, there its contribution is hidden by that from the central star. Most of the radiation longward of a millimeter comes from the outer, optically thin part of the disk. This accounts for the drop of the SED below the extrapolation of the $n=4 / 3$ power law.

\subsubsection{Hydrostatic Equilibrium}

Now we investigate the disk model in which both vertical hydrostatic equilibrium and radiative transfer are treated in a self-consistent fashion. The flaring geometry is governed

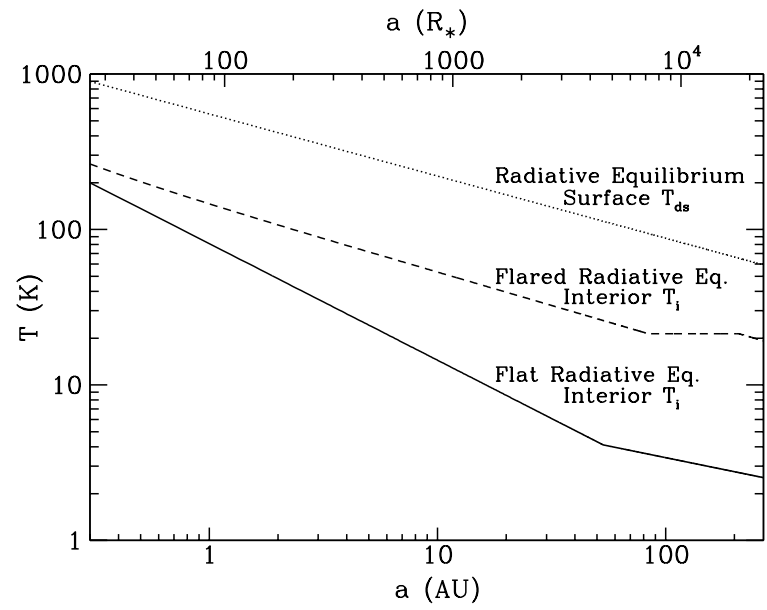

Fig. 4.-Temperature profiles of the flat and flared radiative equilibrium models. The dust temperature $T_{\mathrm{ds}}$ of the superheated layer is independent of disk geometry. Expressions for $T_{d s}$ and $T_{i}$ are provided in the text. The flat disk is truncated at $a_{o}=270 \mathrm{AU}$ (to facilitate comparison with the flared models), before the third temperature regime is reached. 


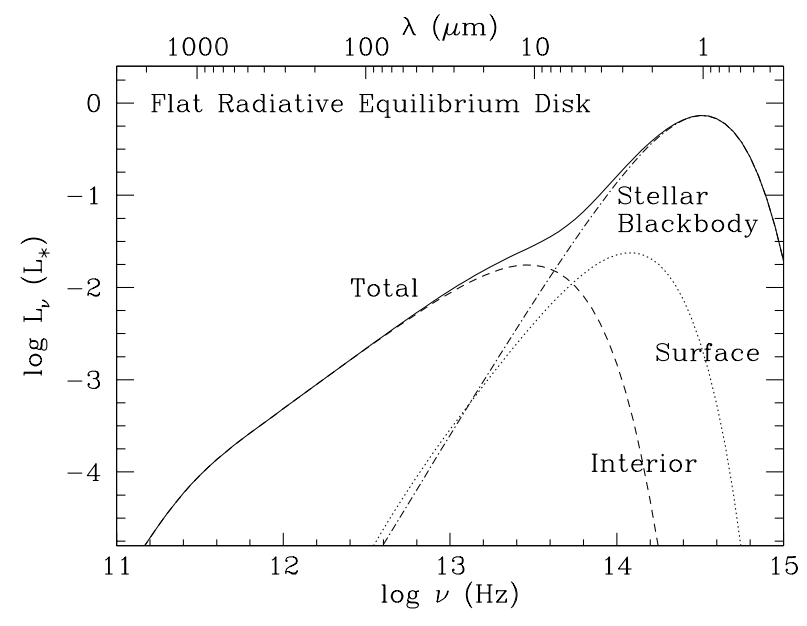

FIG. 5.-SED for the flat radiative equilibrium disk. Emission from the superheated surface is hidden by central starlight. For $30 \lesssim \lambda(\mu \mathrm{m}) \lesssim 1000$, optically thick emission from the disk interior resembles that from the flat blackbody disk (see Fig. 1) but is reduced by a factor of 2 . Emission longward of $1 \mathrm{~mm}$ is optically thin.

by equations (5)-(9). In the limit where the grazing angle is dominated by the flaring term and the disk is opaque to its thermal radiation, the expression for $H / a$ is nearly identical to that given by equation (10). In other, more optically thin regimes, the flaring geometry changes slightly as $T_{i}$ takes successively different forms.

A plot of $T_{i}$ for the flared, radiative equilibrium disk is included in Figure 4. Approximate fitting formulae for $T_{i}$ and $H / a$ in the three regions of the flared, radiative equilibrium disk are as follows. For $0.4<a_{\mathrm{AU}}<84$,

$$
\begin{aligned}
T_{i} & \approx \frac{150}{a_{\mathrm{AU}}^{3 / 7}} \mathrm{~K}, \\
H / a & \approx 0.17 a_{\mathrm{AU}}^{2 / 7} ;
\end{aligned}
$$

for $84<a_{\mathrm{AU}}<209$,

$$
\begin{gathered}
T_{i} \approx 21 \mathrm{~K}, \\
\frac{H}{a} \approx 0.59\left(\frac{a_{\mathrm{AU}}}{84}\right)^{1 / 2} ;
\end{gathered}
$$

and for $209<a_{\mathrm{AU}}<270$,

$$
\begin{aligned}
& T_{i} \approx 21\left(\frac{209}{a_{\mathrm{AU}}}\right)^{19 / 45} \mathrm{~K}, \\
& \frac{H}{a} \approx 0.92\left(\frac{a_{\mathrm{AU}}}{209}\right)^{13 / 45} .
\end{aligned}
$$

The SED for the flared disk truncated at $a_{\mathrm{o}} \approx 270 \mathrm{AU}$, as computed from equation (13), is shown in Figure $6 .^{2}$ Radiation emitted by the surface mirrors that of the interior but is shifted toward, and dominates at, shorter wavelengths. The near power-law rises of both disk components of the SED in the mid-infrared are readily obtained following the procedure used in the derivation of equation (3). The super-

\footnotetext{
${ }^{2}$ The disk may terminate in a slow thermal wind since $H / a \approx 1$ at $a_{\mathrm{AU}}=270$.
}

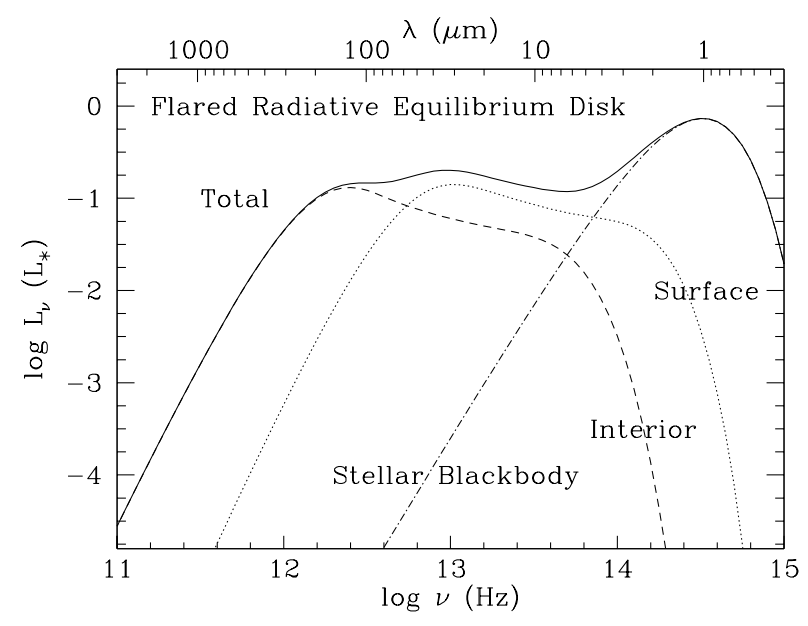

FIG. 6.-SED for the hydrostatic, radiative equilibrium disk. At mid-IR wavelengths, the superheated surface radiates approximately $2-3$ times more power than the interior. Longward of $300 \mu \mathrm{m}, n$ gradually steepens from about 3 to $3+\beta$ as the disk becomes increasingly optically thin.

heated layer obeys the scaling relation $L_{v, s} \sim$ $8 \pi a^{2} \tau_{\mathrm{IR}} \sigma T_{d s}^{4} \sim \alpha L_{*} / 2 \sim 0.06\left(v / 10^{13} \mathrm{~Hz}\right)^{-5 / 7} L_{*}$. Similarly, the opaque interior obeys $L_{v, i} \sim 8 \pi a^{2} \sigma T_{i}^{4} \sim \alpha L_{*} / 2 \sim$ $0.02\left(v / 10^{13} \mathrm{~Hz}\right)^{-2 / 3} L_{*}$. That the former exceeds the latter at fixed wavelength reflects the fact that compared with $L_{v, i}$, most of the contribution to $L_{v, s}$ arises from larger radii and hence larger $\alpha$.

\section{EXTENSIONS AND REFINEMENTS}

\subsection{Planetary Atmosphere Analogy}

Planetary atmospheres are generally transparent to visible radiation above their uppermost cloud decks. ${ }^{3}$ Visible radiation is absorbed by particles. Collisions maintain thermal equilibrium between the gas molecules and the particles. Unit optical depth in the thermal infrared is reached above the cloud tops. Pressure-broadened molecular lines are the main source of infrared opacity. ${ }^{4}$ The total infrared luminosity, the sum of absorbed sunlight and internal heat loss, is emitted as approximate blackbody radiation. Thus, the energy balance of a planetary atmosphere resembles that of the blackbody disk. ${ }^{5}$ This analogy probably accounts for the casual acceptance of the blackbody disk model.

However compelling, this analogy is flawed. T Tauri disks differ from planetary atmospheres in a crucial parameter, the vertical gravitational acceleration. For disks, $g_{\text {disk }} \approx \Omega^{2} z$, with $\Omega$ the orbital angular velocity, and $z$ the distance from the midplane. For our standard parameters, $g_{\text {disk }} \approx 0.4 z_{\mathrm{AU}} a_{\mathrm{AU}}^{-3} \mathrm{~cm} \mathrm{~s}^{-2}$, much smaller than $g_{\text {planet }} \sim 10^{3}$ $\mathrm{cm} \mathrm{s}^{-2}$ for $a \gtrsim a_{i}$. Three consequences of the low vertical gravitational acceleration in disks that invalidate the analogy with planetary atmospheres are described below.

\footnotetext{
${ }^{3}$ Column densities above the cloud tops are similar to that of the minimum-mass solar nebula at $a_{\mathrm{AU}} \approx 1, \Sigma \sim 10^{3} \mathrm{~g} \mathrm{~cm}^{-2}$.

${ }^{4}$ Collision-induced dipole transitions of molecular hydrogen dominate in the atmospheres of the outer planets.

${ }^{5}$ The analogy even extends to the association of the planet's net luminosity with the accretion luminosity of the disk.
} 
Dust grains settle slowly in disks. The gravitational settling time to the midplane,

$$
t_{\text {settle }} \approx \frac{\Sigma}{r \rho_{d} \Omega} \approx 10^{7}\left(\frac{0.1 \mu \mathrm{m}}{r}\right)\left(\frac{\Sigma_{0}}{10^{3} \mathrm{~g} \mathrm{~cm}^{-2}}\right) \mathrm{yr},
$$

is independent of $a$. It is longer than the disk lifetime for particles that most efficiently absorb stellar radiation. Moreover, the settling may be slowed by turbulent mixing or vertical circulation of the gas.

The gas pressure in disks is so low that dust grains directly exposed to stellar radiation reradiate almost all of the stellar energy they absorb. Thus, the temperature of the superheated grains is determined by radiative balance and is independent of the ambient gas temperature. The regulation of the gas temperature is a more subtle issue (cf. § 3.2).

The low pressure in disks also means that molecular lines suffer negligible pressure broadening. Thus, the gas opacity is concentrated in narrow, Doppler-broadened lines. Important molecular sources of opacity must have permitted dipole transitions near the peak of the blackbody spectrum corresponding to the ambient temperature $T$. Furthermore, these transitions must connect energy levels that do not lie too far above $k T$. Water is a prime candidate for a coolant at temperatures greater than $100 \mathrm{~K}$. It is abundant, and as a consequence of being both a hydride and an asymmetric rotor, it possesses a rich rotation spectrum shortward of $300 \mu \mathrm{m}$. However, our crude estimates suggest that even if much of the cosmic abundance of oxygen is tied up in water, water lines would not cover more than a small fraction of the infrared spectrum at any temperature.

\subsection{Energy Balance in the Superthermal Dust Layer}

Stellar energy absorbed by dust grains is lost through emission of infrared radiation and collisional transfer to gas molecules. The gas molecules, mostly $\mathrm{H}_{2}$ and $\mathrm{He}$, gain energy in collisions with dust grains and lose it in collisions with molecular coolants such as $\mathrm{H}_{2} \mathrm{O}$. The energy added to the internal degrees of freedom of the coolants in collisions with gas molecules and by absorption of dust radiation is lost in radiation to space. Thus, $T_{d s} \gtrsim T_{g s} \gtrsim T_{x}$, where the subscripts $j=\{d s, g s, x\}$ denote quantities pertaining to the dust, the inert gas molecules, and the molecular coolants, respectively. ${ }^{6}$

Balancing the rates of energy gain and loss per unit surface area of a dust grain yields

$$
\sigma T_{*}^{4}\left(\frac{R_{*}}{a}\right)^{2} \approx \epsilon_{s} \sigma T_{d s}^{4}+n_{g} v_{g} k\left(T_{d s}-T_{g s}\right),
$$

with $v_{g}$ being the sound speed. For $n_{g} \ll n_{c 1}$, where the critical number density

$$
n_{c 1} \approx \frac{\epsilon_{s} \sigma T_{d s}^{3}}{v_{g} k} \approx \frac{\sigma \mu_{g}^{1 / 2} T_{*}^{5 / 2}}{k^{3 / 2}}\left(\frac{R_{*}}{a}\right)^{7 / 5} \approx \frac{10^{14}}{a_{\mathrm{AU}}^{7 / 5}} \mathrm{~cm}^{-3},
$$

gas-grain collisions have a negligible effect on $T_{d s}$, and equation (16) reduces to equation (11). ${ }^{7}$ On the other hand, for $n_{g} \gg n_{c 1}, T_{d s} \approx T_{g s}$. The gas density in the superheated dust layer, $n_{g s}$, is unknown. It could be as large as $n_{0}$, the value at the disk midplane, or as low as $n_{\min }$, the value obtained by assuming uniform mixing of dust and gas up to

\footnotetext{
${ }^{6}$ The symbol $T_{\mathrm{x}}$ refers to the excitation temperature of the coolant transition.

${ }^{7}$ We evaluate $v_{g}$ on the right-hand side of eq. (17) at temperature $T_{d s}$.
}

the tenuous outer layers of the disk. Runs of $n_{c 1}, n_{0}$, and $n_{\min }$ as functions of $a$ are displayed in Figure 7. It is seen that even $n_{0}$ falls below $n_{c 1}$ beyond $a_{\mathrm{AU}} \approx 2$.

Balancing the rates at which inert gas molecules gain and lose energy in collisions with dust grains and molecular coolants yields

$$
n_{d s} \sigma_{d}\left(T_{d s}-T_{g s}\right) \approx n_{x} \sigma_{x}\left(T_{g s}-T_{x}\right)
$$

where $n$ is a number density, and $\sigma$ denotes a collisional cross section. The compositions and mixing ratios of molecular coolants are uncertain. Photodissociation and incorporation into dust grains represent loss mechanisms that can reduce abundance ratios far below values based on cosmic abundances. Nevertheless, let us suppose that the mass in coolant molecules is comparable to that in dust grains. Then $n_{d s} \sigma_{d} / n_{x} \sigma_{x} \sim 10^{-3}$, the ratio of the linear size of a gas molecule to a dust grain. Thus, we would not be surprised to discover that $T_{g s}-T_{x} \ll T_{d s}-T_{g s}$.

Estimating excitation temperatures for the transitions in molecular coolants is a daunting task. We proceed by making a number of drastic approximations. Each transition is assumed to give rise to an optically thick, Dopplerbroadened line having excitation temperature $T_{x}$. For clarity, we begin by considering the pure radiative excitation of these levels. It is not difficult to show that the excitation comes mainly from radiation emitted by the superheated dust grains as opposed to that from the interior portion of the disk. We specialize further to transitions whose frequencies lie on the Rayleigh-Jeans part of the blackbody spectrum at temperature $T_{d s}$. Applying the basic concepts of the escape probability formalism for radiative transfer to a two-level molecule, we obtain

$$
\begin{aligned}
& T_{x} \approx \alpha \epsilon_{s} T_{d s} \text { for } h v \lesssim k T_{x}, \\
& T_{x} \approx \frac{h v}{k} \ln \left(\frac{1}{\alpha \epsilon_{s}} \frac{h v}{k T_{d s}}\right) \text { for } h v \gtrsim k T_{x} .
\end{aligned}
$$

Pure radiative excitation sets a floor on the value of $T_{x}$.

Next we consider the effects of collisions while neglecting radiative excitation. Here we suppose that lines of bright-

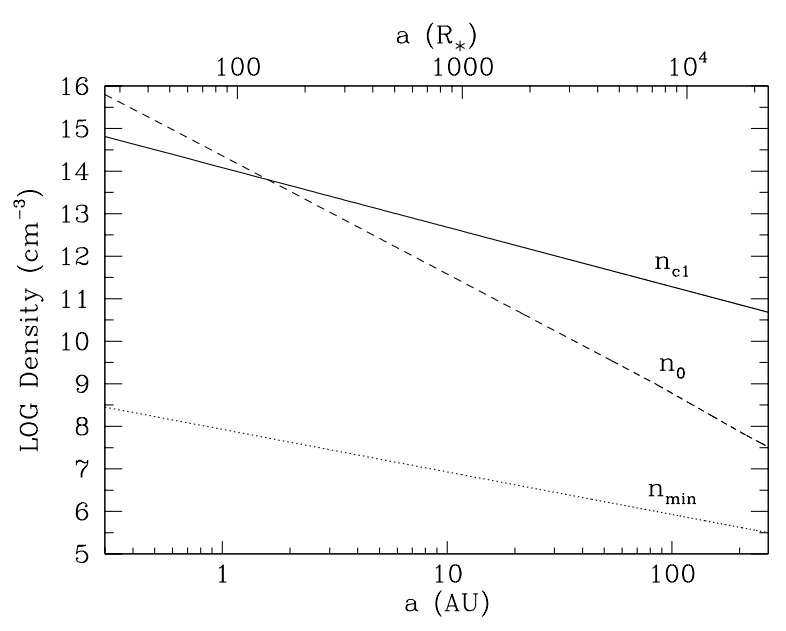

FIG. 7.-Comparison of possible gas densities in the superheated layer with the critical density $n_{c 1}$ above which gas-grain collisions might lower the dust temperature. The actual gas density could be as high as $n_{0}$, the value at the disk midplane, or as low as $n_{\min }$, the value for a disk in which gas and dust are uniformly mixed everywhere. Except in the innermost 2 $\mathrm{AU}$, it is evident that the dust likely remains superheated at the temperature given by eq. (11). 
ness temperature $T_{x}$ cover a fraction $f$ of the blackbody spectrum at temperature $T_{x}$. We equate the emission-line flux to the rate (per unit disk surface area) at which the gas gains energy in collisions with the superheated dust grains:

$$
f \sigma T_{x}^{4} \approx n_{g s} v_{g} k \alpha\left(T_{d s}-T_{g s}\right) .
$$

For $n_{g s} \ll n_{c 2}$, where the second critical gas density

$$
n_{c 2} \approx{\frac{f}{\alpha \epsilon_{s}}}_{n_{c 1}}
$$

we find

$$
T_{x} \approx\left(\frac{n_{g s}}{n_{c 2}}\right)^{1 / 4} T_{d s} .
$$

In the high-density limit $n_{g s} \gg n_{c 1}, T_{x} \approx T_{g s} \approx T_{d s} \approx T_{s}$, with

$$
T_{s} \approx\left(\frac{\alpha}{f+\alpha \epsilon_{s}}\right)^{1 / 4}\left(\frac{R_{*}}{a}\right)^{1 / 2} T_{*} .
$$

If $f \ll \alpha \epsilon_{s}$, equation (22b) reduces to equation (11), the appropriate expression for the dust temperature in the absence of cooling by molecular line emission. On the other hand, if $f \gg \alpha \epsilon_{s}$, equation (22b) collapses to the expression appropriate for a blackbody disk as given by equation (1), magnified by a factor of about $1 / f^{1 / 4}$.

\subsection{Location of the Superthermal Layer}

We have been assuming that the dust and gas are uniformly mixed throughout the disk. Consequently, for our model, the gas density is much smaller in the superthermal dust layer than in the disk midplane (cf. Fig. 7). Although the timescale for dust grains to settle to the disk midplane may be long compared with the disk lifetime (cf. eq. [15]), the timescale for grains to fall through the superthermal layer (in the absence of advection by the gas) is considerably shorter. The latter is given by

$$
t_{\text {settle }, s} \sim \frac{v_{g}}{a r \kappa_{V} \rho_{d} \Omega^{2}} \sim a_{\mathrm{AU}}^{2} \mathrm{yr} .
$$

Thus, the superthermal dust layer is likely to be found closer to the disk midplane and to have a lower $\kappa_{V}$ than our simple assumption of uniform dust-gas mixing implies. ${ }^{8}$

How might we learn about the level at which the superthermal dust layer sits? One possibility is through the observation of emission lines that would form in the gas associated with this layer if the gas temperature is comparable to the dust temperature. Perhaps most revealing would be observations of the rotation lines of $\mathrm{H}_{2}$. Because these arise from quadrupole transitions and are therefore weak, they would only be seen if the dust layer were located where the gas density is much larger than the minimum value given by the assumption of uniform mixing of dust and gas.

\subsection{Dust Albedo}

A nonzero dust grain albedo, $A_{d}$, at visible wavelengths would reduce the absorbed stellar flux by a factor of order

\footnotetext{
${ }^{8}$ The timescale for dust collisions/coagulation to occur in the superthermal layer is $1 / \alpha$ times larger than $t_{\text {settle }, s}$.
}

$\left(1-A_{d}\right)^{1 / 2}$. This would lower the disk thickness, but by a less impressive factor of order $\left(1-A_{d}\right)^{1 / 16}$.

How small might $\left(1-A_{d}\right)^{1 / 2}$ be? Values of $A_{\mathrm{d}} \sim 0.4-0.5$ are typical of interstellar grains (Draine $\&$ Lee 1984). Visual albedos of pure ice particles are much closer to unity. And ices are likely to comprise a significant fraction of the mass in grains in the outer disk. Starting inside $a_{\mathrm{AU}}=10^{2}$, the partial pressures of $\mathrm{H}_{2} \mathrm{O}$, then $\mathrm{NH}_{3}$, and finally $\mathrm{CH}_{4}$ are expected to exceed their vapor pressures. However, taking the outer planet atmospheres as a guide, it seems unlikely that any ice so formed would be pure enough to yield $\left(1-A_{d}\right)^{1 / 2} \lesssim 0.3$.

\subsection{Accretional Heating}

A star that derives its radiant energy from accretion has $T_{*} \approx\left(G M_{*} \dot{M}_{*} / R_{*}^{3} \sigma\right)^{1 / 4}$, where $\dot{M}_{*}$ is the mass accretion rate (provided the accretional energy is radiated over the entire stellar surface). The stellar flux impinging on the circumstellar disk, $\sigma T_{*}^{4}\left(R_{*} / a\right)^{2} \alpha$, is to be compared with the local viscous dissipation rate per unit area, $\sigma T_{*}^{4}\left(R_{*} / a\right)^{3}$. Thus, in the extreme case where accretion accounts for the entire stellar luminosity, viscous dissipation is only competitive with stellar heating for $a \lesssim a_{\mathrm{tr}}$.

The bulk of the viscous dissipation probably takes place deep inside the disk. Provided the disk is sufficiently opaque, this could result in midplane temperatures being higher than $T_{*}\left(R_{*} / a\right)^{3 / 4}$ by a factor of order $\tau^{1 / 4}$, where $\tau \approx \epsilon_{i} \kappa_{V} \Sigma$ is the effective vertical optical depth. A concomitant increase in the disk thickness by a factor of $\tau^{1 / 8}$ changes the surface geometry and may cause some portions of the disk to be shadowed from direct exposure to starlight. Such effects are likely to be important only for the innermost regions of the disk where radiation temperatures, surface densities, and consequently optical depths are highest. For example, around an accretion-powered star, the midplane temperature of our standard disk is about $2000 \mathrm{~K}$ at $a_{\mathrm{AU}}=$ 1.5. This yields about a factor 2 increase in disk thickness relative to that of the passive disk. For smaller radii, the enhanced thickness would be buffered somewhat by the reduction of opacity associated with the vaporization of silicates.

\section{DISCUSSION}

\subsection{Comparison with Observations}

\subsubsection{Continuum SEDs}

In Figure 8, we demonstrate that the nearly flat infrared excess of the classical T Tauri star GM Aur can be naturally interpreted as arising from a passive reprocessing disk. Parameters for the central star are obtained from Beckwith et al. (1990), and a distance of $140 \mathrm{pc}$ to the Taurus-Auriga cloud is assumed. Approximate best-fit parameters for the assumed face-on disk are the same as those for our fiducial model, with the following alterations: $\beta=1.4, r_{p}=0.3 \mu \mathrm{m}$, $\Sigma_{0}=3 \times 10^{3} \mathrm{~g} \mathrm{~cm}^{-2}, a_{o}=4 \times 10^{4} R_{*}=390 \mathrm{AU}$, and $a_{i}=$ $800 R_{*}=6.8 \mathrm{AU}$.

The inner cutoff radius is chosen so that the model matches the observationally determined SED at $12 \mu \mathrm{m}$; a smaller cutoff radius results in excessive emission. However, it seems likely that the central hole is an artifact of fitting a face-on model to observations of a disk viewed at a nonzero inclination angle, $i$. Millimeter-wave aperture synthesis images in CO and Hubble Space Telescope (HST) images in scattered stellar light reveal a flared circumstellar disk in 


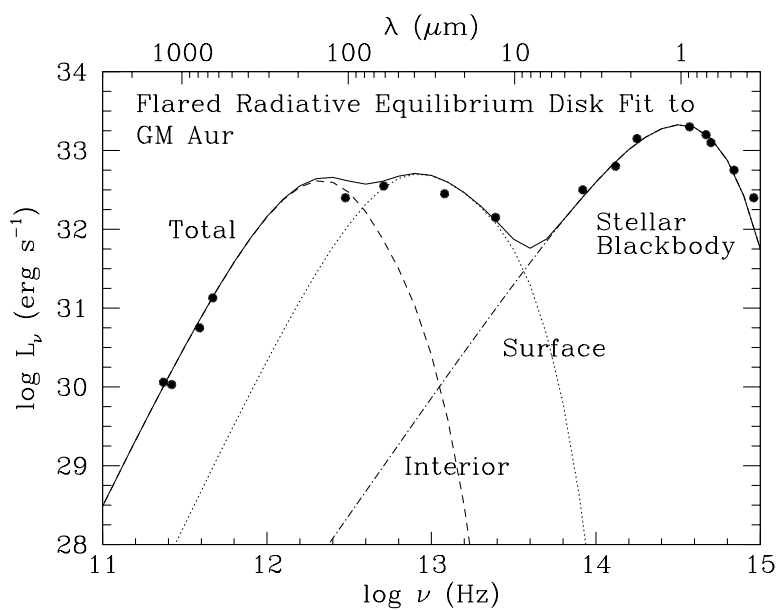

FIG. 8.-Observed SED of GM Aur ( filled circles) and accompanying hydrostatic, radiative equilibrium (face-on) disk model. Fit parameters are as follows: $\Sigma=3 \times 10^{3} a_{\mathrm{AU}}^{-3 / 2} \mathrm{~g} \mathrm{~cm}^{-2}, \beta=1.4, r_{\mathrm{p}}=0.3 \mu \mathrm{m}, a_{o}=390 \mathrm{AU}$, and $a_{i}=6.8 \mathrm{AU}$. The derived inner cutoff radius is likely an artifact of fitting a face-on model to a disk observed at substantial inclination (see the text for a discussion).

Keplerian rotation (Koerner 1997) and suggest that $i \sim 60^{\circ}$. A cos $i$ correction factor applied to emission from the optically thick interior does not change the fitted SED noticeably. However, the flared outer "wall" may absorb radiation emitted toward Earth from the inner disk, thereby mimicking in the SED the signature of a central hole in a disk viewed face-on. We reserve a more detailed discussion of the effects of nonzero inclination angle for a future study.

\subsubsection{Spatially Resolved Broadband Observations}

Pioneering observations that marginally resolve disks at millimeter wavelengths are reported in Lay et al. (1994) and Mundy et al. (1996). Fits of elliptical Gaussians to the $\lambda=0.87 \mathrm{~mm}$ brightness distributions of HL Tau and L1551 IRS 5 yield semimajor radii at half-maximum brightness of 60 and $80 \mathrm{AU}$, respectively (Lay et al. 1994). Similar results were obtained at $\lambda=2.7 \mathrm{~mm}$ for HL Tau, with the semiminor radius marginally resolved to be $\sim 30 \mathrm{AU}$ (Mundy et al. 1996).

Interferometric observations of the nearest $\mathrm{T}$ Tauri disks at $\lambda \approx 10 \mu \mathrm{m}$ with 10 mas resolution might separate the contributions to the SED from the superthermal dust layer and the disk interior. Running integrals for $L_{v, s}$ and $L_{v, i}$ against $a$ are displayed in Figure 9. Most of the $10 \mu \mathrm{m}$ emission inward of $1 \mathrm{AU}$ originates from the disk interior, whereas the contribution from the superthermal layer is localized in an annulus centered on $\sim 10$ AU. Similar results hold for the $20 \mu \mathrm{m}$ flux. At wavelengths longer than $\sim 100 \mu \mathrm{m}$, the disk interior dominates the emission.

\subsubsection{Spectral Dust Features}

Dust grains possess spectral resonances. Among the best known are those at $\lambda=9.7$ and $18 \mu \mathrm{m}$, which are thought to arise from silicates (see, e.g., Draine 1995); the former has been observed in both emission and absorption in the spectra of T Tauri stars. For example, Cohen \& Witteborn (1985) identified $10 \mu \mathrm{m}$ emission features in 24 stars and 10 $\mu \mathrm{m}$ absorption features in another seven in a spectrophotometric survey of $32 \mathrm{~T}$ Tauri stars. The emission features evince line-to-continuum ratios of about 1.2-3.0 to 1 .

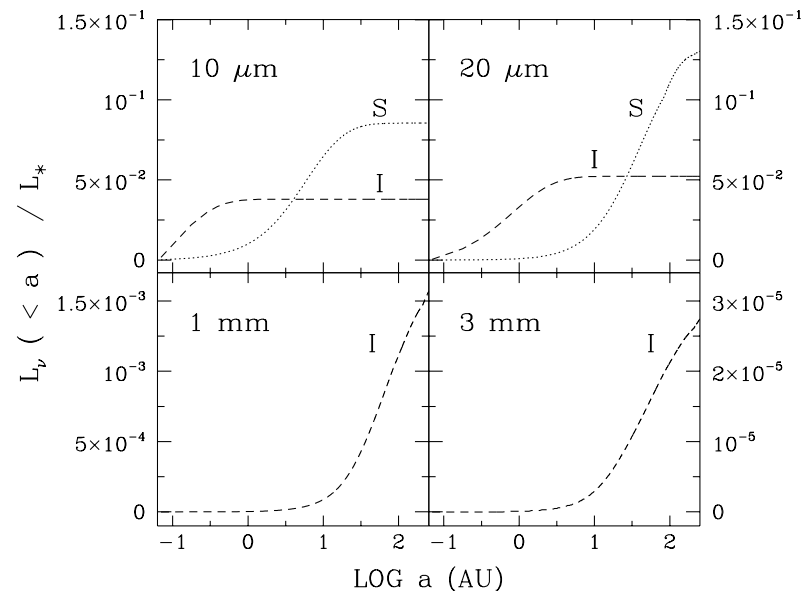

FIG. 9.- Running contributions to the SED from the superthermal dust layer (S) and the disk interior (I) at wavelengths for which the disk may be increasingly resolved by interferometers. For a given disk component, $L_{v}(<a)$ is given by eq. (13), with $a_{o}$ replaced by $a$. At $\lambda=1$ and $3 \mathrm{~mm}$, the contributions from the surface layer are negligible and not shown.

Emission features are a natural consequence of the superheated dust layer for disks viewed nearly face-on. Line-tocontinuum ratios should be of the same order as the percentage increase in dust emissivity associated with the spectral feature. To illustrate the effects of spectral resonances, we have employed an emissivity profile motivated by the data for "outer-cloud dust" as given by Mathis (1990). The resulting (face-on) SED displayed in Figure 10 exhibits emission features at 10 and $20 \mu \mathrm{m}$.

Absorption features may be associated with disks viewed nearly edge-on. Support for this hypothesis comes from two observational findings. These features appear most prominently among the "extreme" flat spectrum sources for which $L_{v}$ at infrared wavelengths matches or exceeds $L_{v}$ in the visible. There is a positive correlation between the strengths of these features and the fractional linear polarization at optical wavelengths. Both findings are plausibly the

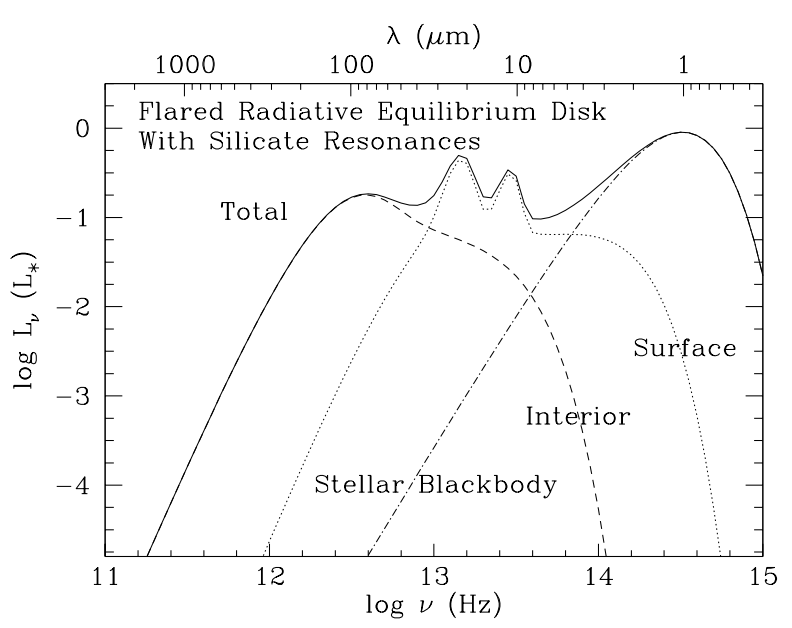

FIG. 10.-SED for the hydrostatic, radiative equilibrium disk using a grain emissivity profile motivated by data from Mathis (1990). For wavelengths shorter than $0.3 \mu \mathrm{m}$, our assumed emissivity is unity; longward of $0.3 \mu \mathrm{m}$, it obeys a (single) power-law relation $\epsilon_{\lambda}=(0.3 \mu \mathrm{m} / \lambda)^{1.4}$, on which are superposed two Gaussians centered on 10 and $20 \mu \mathrm{m}$, having amplitudes that are 3 times their local continuum emissivity and FWHM equal to 3 and $9 \mu \mathrm{m}$, respectively. 
consequence of the extinction of optical radiation in a flared disk viewed at large inclination angle.

\subsubsection{Temporal Behavior}

Temporal variations would provide another diagnostic of circumstellar disks. The claim by Moriarty-Schieven \& Butner (1997) that the submillimeter and millimeter fluxes from the T Tauri binary GG Tau increased by factors of order 2 between 1992 and 1994 is of relevance here. The cause of this "radio-wave flare" has not been identified. A plausible hypothesis is that it resulted from enhanced disk heating associated with a burst in luminosity originating near one component, or both components, of the central binary. This leads us to consider relevant timescales for the radiative and hydrostatic response of the disk.

Seven different timescales come into play. They are (1) the timescale over which superheated dust grains in the surface layer equilibrate with the ambient stellar radiation field,

$$
t_{d s} \sim \frac{r \rho_{d} k T_{d s} a^{2}}{\mu_{d} R_{*}^{2} \sigma T_{*}^{4}} \sim 0.02 a_{\mathrm{AU}}^{8 / 5} \mathrm{~s},
$$

where $\mu_{d} \approx 10 \mu_{g}$ is the mean molecular weight per degree of freedom in a dust grain; (2) the light-travel timescale from star to disk,

$$
t_{1 \mathrm{t}} \sim \frac{a}{c} \sim 5 \times 10^{2} a_{\mathrm{AU}} \mathrm{s} ;
$$

(3) the photon diffusion timescale through the disk, ${ }^{9}$

$$
t_{\mathrm{diff}} \sim \frac{\epsilon_{i} \kappa_{V} \Sigma h}{c} \sim \frac{2 \times 10^{5}}{a_{\mathrm{AU}}^{9 / 14}} \mathrm{~s}
$$

(4) the thermal timescale for the dust if it were decoupled from the gas, ${ }^{10}$

$$
\begin{aligned}
t_{d i} \sim & \frac{\Sigma_{d} k}{\mu_{d} \sigma T_{i}^{3}}\left(1+\frac{1}{\epsilon_{i} \kappa_{V} \Sigma}\right) \sim \frac{2 \times 10^{5}}{a_{\mathrm{AU}}^{3 / 14}} \mathrm{~s} \\
& \times \begin{cases}\left(1+10^{-4} a_{\mathrm{AU}}^{2714}\right) & \text { for } a_{\mathrm{AU}} \lesssim 84, \\
{\left[1+\left(\frac{a_{\mathrm{AU}}}{84}\right)^{3 / 2}\right]} & \text { for } 84 \lesssim a_{\mathrm{AU}} \lesssim 270,\end{cases}
\end{aligned}
$$

where $\Sigma_{d} \approx 10^{-2} \Sigma$ is the surface density of dust; (5) the thermal timescale for the gas, ${ }^{10}$

$$
\begin{aligned}
t_{g i} \sim & \frac{\Sigma k}{\mu_{g} \sigma T_{i}^{3}}\left(1+\frac{1}{\epsilon_{i} \kappa_{V} \Sigma}\right) \sim \frac{2 \times 10^{8}}{a_{\mathrm{AU}}^{3 / 14}} \mathrm{~s} \\
& \times \begin{cases}\left(1+10^{-4} a_{\mathrm{AU}}^{2714}\right) & \text { for } a_{\mathrm{AU}} \lesssim 84, \\
{\left[1+\left(\frac{a_{\mathrm{AU}}}{84}\right)^{3 / 2}\right]} & \text { for } 84 \lesssim a_{\mathrm{AU}} \lesssim 270 ;\end{cases}
\end{aligned}
$$

\footnotetext{
${ }^{9}$ This timescale is only relevant where the disk interior is opaque to its thermal radiation.

${ }^{10}$ In evaluating the timescale at $a_{\mathrm{AU}} \gtrsim 84$, we have set $T_{i}=21 \mathrm{~K}$.
}

(6) the timescale for the dust temperature to relax to the gas temperature,

$$
t_{\text {relax }} \sim \frac{r \rho_{d}}{\mu_{d} n_{g} v_{g}} \sim \frac{\mu_{g} r \rho_{d}}{\mu_{d} \Sigma \Omega} \sim 10^{-2} a_{\mathrm{AU}}^{3} \mathrm{~s} ;
$$

(7) the dynamical timescale over which the disk adjusts to departures from hydrostatic equilibrium,

$$
t_{\mathrm{dyn}} \sim 1.4 a_{\mathrm{AU}}^{3 / 2} \mathrm{yr} .
$$

How rapidly might the SED vary in response to changes in the luminosity of the central star? Since $t_{d s} \ll t_{1 \mathrm{t}}$, contributions from the surface layer are limited by $t_{1 \mathrm{t}}$. Those from the interior are limited by $t_{g i}$, since $t_{\text {relax }} \lesssim t_{d i}$ and $t_{\text {diff }} \ll$ $t_{g i} \cdot{ }^{11}$ To relate these response times to timescales for variation at a fixed wavelength, $\lambda$, consult Figure 9.

\subsection{Unresolved Issues}

Our investigation leaves many unresolved issues.

Is a disk in radiative and hydrostatic equilibrium dynamically stable?

How does the SED depend upon disk inclination?

How much of the thermally emitted spectrum is covered by molecular lines? Do the lines appear in absorption or emission? Which molecules are they associated with?

How high does the layer of superthermal dust grains sit above the disk midplane? What is the gas temperature in this layer? Is gravitational settling of dust grains seriously impeded by turbulent mixing or by vertical circulation? Or does radiation pressure from the central star dominate all the forces, so that the superthermal grains are driven into denser environments and the disk geometry flattens?

What is the albedo of the circumstellar dust? How does scattered stellar radiation compare with thermal emission in the near-IR?

Does active accretion significantly affect the SEDs, either by preventing the settling of dust grains or by thickening the inner regions of the disk?

We thank Anneila Sargent for providing the data on GM Aur and other T Tauri stars, Shri Kulkarni for suggesting that temporal variability of disk emission can provide a further means of probing disk characteristics, Dave Koerner and Karl Stapelfeldt for informative discussions regarding GM Aur, and Steve Beckwith for a thorough and thoughtful referee's report. Financial support for this research was provided by NSF grant 94-14232. E. C. acknowledges support from an NSF Graduate Fellowship.

${ }^{11}$ The former inequality is only marginally satisfied at $a_{o}$.

\section{REFERENCES}

Adams, F. C., Lada, C. J., \& Shu, F. H. 1987, ApJ, 312, 788

Adams, F. C., Ruden, S. P., \& Shu, F. H. 1989, ApJ, 347, 959

Beckwith, S. V. W., Sargent, A. I., Chini, R. S., \& Gusten, R. 1990, AJ, 99, 924

Calvet, N., Hartmann, L., Kenyon, S. J., \& Whitney, B. A. 1994, ApJ, 434, 330

Cohen, M., \& Witteborn, F. C. 1985, ApJ, 294, 345

\footnotetext{
Draine, B. T. 1995, in ASP Conf. Ser. 80, The Physics of the Interstellar Medium and Intergalactic Medium, ed. A. Ferrara, C. Heiles, C. McKee, \& P. Shapiro (San Francisco: ASP), 133

Draine, B. T., \& Lee, H. M. 1984, ApJ, 285, 89

Kenyon, S. J., \& Hartmann, L. 1987, ApJ, 323, 714

Koerner, D. W. 1997, Origins of Life and Evolution of the Biosphere, 27, 157
} 
Kusaka, T., Nakano, T., \& Hayashi, C. 1970, Prog. Theor. Phys., 44, 1580

Lay, O. P., Carlstrom, J. E., Hills, R. E., \& Phillips, T. G. 1994, ApJ, 434, L75

Lynden-Bell, D., \& Pringle, J. E. 1974, MNRAS, 168, 603

Mathis, J. M. 1990, ARA\&A, 28, 37

McCaughrean, M. J., \& O’Dell, C. R. 1996, AJ, 111, 1977

Mendoza, E. E. V. 1968, ApJ, 151, 977

Moriarty-Schieven, G. H., \& Butner, H. M. 1997, ApJ, 474, 768

Mundy, L. G., et al. 1996, ApJ, 464, L169

Natta, A. 1993, ApJ, 412, 761

Ostriker, E. C., Shu, F. H., \& Adams, F. C. 1992, ApJ, 399, 192
Rucinski, S. M. 1985, AJ, 90, 2321

Ruden, S. P., \& Pollack, J. B. 1991, ApJ, 375, 740

Rydgren, A. E., Schmelz, J. T., Zak, D. S., \& Vrba, F. J. 1984, Broad Band Spectral Energy Distributions of $\mathrm{T}$ Tauri Stars in the Taurus-Auriga Region (Washington, DC: US Naval Obs.), 2d Ser. Vol. 25, 1

Rydgren, A. E., \& Zak, D. S. 1987, PASP, 99, 141

Shu, F. H., Adams, F. C., \& Lizano, S. 1987, ARA\&A, 25, 23

Shu, F. H., Tremaine, S., Adams, F. C., \& Ruden, S. P. 1990, ApJ, 358, 495

Strom, K. M., Strom, S. E., Edwards, S., Cabrit, S., \& Skrutskie, M. F. 1989, AJ, 97,1451

Weidenschilling, S. J. 1977, Ap\&SS, 51, 153 